5. L. J. Comrie, "Construction of tables by interpolation," Royal Astr. Soc., Monthly Notices, v. 88, 1928 , p. 506-523.

6. J. C. P. MILLER, op. cit., p. 196. 1954.

7. MASSAChUSETTS INSTITUTE of Technology, Mathematical Tables Symposium, Cambridge,

8. NBS Applied Mathematics Series No. 9, Tables of Chebyshev Polynomials $S_{n}(x)$ and $C_{n}(x)$, U. S. Govt. Printing Office, Washington, 1952.

9. C. W. Clenshaw \& F. W. J. Olver, "The use of economical polynomials in mathematical tables," Cambridge Phil. Soc., Proc., 51, 1955, p. 614-628.

Note added in proof. Dr. L. Fox of the National Physical Laboratory, Teddington, Middlesex, England, has recently informed the author in a paper as yet unpublished, that in fact the Everett form of the modified Chebyshev-Bessel interpolation formula gives slightly better results (in the sense that the maximum possible residual error is smaller) than the formula advocated here.

\title{
Iterative Procedure for Evaluating a Transient ResponseThrough its Power Series
}

Introduction. We discuss here a particular method of evaluating a time function, such as a transient response, from its Laplace transform. We shall assume the Laplace transform of $F(t)$,

$$
\bar{F}(S)=\int_{0}^{\infty} e^{-s t} F(t) d t
$$

is given, and is of the form

$$
\bar{F}(S)=\frac{A_{0}{ }^{0} S^{N-1}+A_{1}{ }^{0} S^{N-2}+\cdots+A^{0}{ }_{N-2} S+A^{0}{ }_{N-1}}{S^{N}+b_{1} S^{N-1}+b_{2} S^{N-2}+\cdots+b_{N-1} S+b_{N}} .
$$

(The superscript 0 of the $A$ 's does not indicate a power, but is used as a superscript for reasons which will appear presently.)

We require in (2) that the denominator be of higher degree than the numerator, which is necessary if $F(t)$ is a Laplace transform. We shall always assume also that we have $\bar{F}(S)$ written with the coefficient of $S^{N}$ in the denominator equal to unity, as in equation (2).

The method described below is an iterative method for obtaining the quantities $\alpha_{j}$ in MacLaurin series for $F(t)$, which we write in the form

$$
F(t)=\sum_{j=0}^{\infty} \alpha_{j} \frac{t^{j}}{j !}
$$

The method has the advantage that it is not necessary to know the roots of the numerator or denominator of $\bar{F}(S)$. The series (3) will converge for all positive values of $t$, although for larger values of $t$ a great many terms may be needed. This, however, is not too great a disadvantage for digital purposes, since the method for computing the $\alpha_{j}$ 's is an iterative one. It is necessary, however, to have some idea of how many terms will be needed, and a criterion for this is also given below and discussed. The theoretical derivations are given in the Appendix.

Method for Computing the $\alpha_{j}$ 's. The method for computing the $\alpha_{j}$ 's, the theory of which is discussed in the Appendix, is as follows: We start with the coefficients

$$
A_{0^{0}}, A_{1}{ }^{0}, \cdots, A^{0}{ }_{N-1}, b_{1}, b_{2}, \cdots, b_{N} \text { of (2). }
$$


Then

$$
\alpha_{0}=A_{0}{ }^{0} \text {. }
$$

We next evaluate the numbers $A_{0}{ }^{1}, A_{2}{ }^{1}, \cdots, A^{1}{ }_{N-1}$ as follows :

(4)

$$
\begin{aligned}
& A_{0}{ }^{1}=A_{1}{ }^{0}-\alpha_{0} b_{1} \\
& A_{1}{ }^{1}=A_{2}{ }^{0}-\alpha_{0} b_{2} \\
& \dot{\cdot} \\
& A^{1}{ }_{N-2}=A^{0}{ }_{N-1}-\alpha_{0} b_{N-1} \\
& A^{1}{ }_{N-1}=-\alpha_{0} b_{N} .
\end{aligned}
$$

Then

Then we evaluate

$$
\alpha_{1}=A_{0}{ }^{1} .
$$

$$
\begin{aligned}
& A_{0}{ }^{2}=A_{1}{ }^{1}-\alpha_{1} b_{1} \\
& A_{1^{2}}{ }^{2}=A_{2}{ }^{1}-\alpha_{1} b_{2}
\end{aligned}
$$

$$
\begin{aligned}
\alpha_{j} & =A_{0^{j}} \\
A_{k}^{j+1} & =A_{k+1}^{j}-\alpha_{j} b_{k+1}, \quad 0 \leq k \leq N-2 \\
A_{N-1}^{j+1} & =-\alpha_{j} b_{N}
\end{aligned}
$$

when $j=0,1,2,3$, etc.

Thus the numbers $\alpha_{j}$ can be obtained and substituted in equation (3) to give $F(t)$.

Number of Terms. It is difficult to estimate the exact number of terms of the series (3) necessary to give a prescribed accuracy. However, it is shown in the Appendix that if we wish to have $F(t)$ accurate to within $\pm \epsilon$ with $0<t \leq t_{0}$, neglecting numerical round-off errors, it is sufficient to have $n>n_{0}$, where $(n+1)$ is the number of terms (counting the 0 order term), and

$$
n_{0}=e(1+b) t_{0}+\ln \left[\frac{A(1+b)\left[(1+b)^{N}-1\right]}{2 b \epsilon(e-1)}\right]
$$

here $A$ is the maximum of the numbers $\left|A_{0}{ }^{0}\right|,\left|A_{1}{ }^{0}\right|, \cdots,\left|A^{0}{ }_{N-1}\right| ; b$ is the maximum of the numbers $\left|b_{1}\right|,\left|b_{2}\right|, \cdots,\left|b_{N}\right|$; and $e$ is the base of the natural logarithms- "ln" denotes the natural logarithm. 
It should be borne in mind that the above criterion may give more terms than are actually necessary in a particular case. However, it has the advantage that it offers a method of determining the number of terms from the function $\vec{F}(S)$ alone, without necessarily having advance knowledge concerning the function $F(t)$.

As an illustration we consider two simple cases where $F(t)$ is easily evaluated by elementary methods. First consider

Then we know

$$
\bar{F}(S)=\frac{1}{S+1} .
$$

$$
F(t)=e^{-t} .
$$

(Our iterative procedure will give $\alpha_{j}=(-1)^{i}$.)

In this case, $A=b=N=1$. If we take $t_{0}=5$ seconds, and $\epsilon=10^{-3}$, substitution in equation (7) gives $n_{0}=31.3$, so we would use 33 terms (counting the 0 order term). However if we take $n=17$, our error is less than $5^{18} / 18$ ! which is less than $10^{-3} \cdot 5^{17} / 17$ ! is greater than $10^{-3}$. Thus the actual number that we need in this case is $n=17$, or 18 terms, so that equation (7) gave us far too many.

Next consider

$$
\bar{F}(S)=\frac{2 S+11}{S^{2}+11 S+10} \quad(N=2, A=b=11) .
$$

Here again we can find directly that

$$
F(t)=e^{-t}+e^{-10 t}
$$

so in this case

$$
\alpha_{j}=(-1)^{i}\left[10^{i}+1\right] .
$$

Substitution in (7), with the same $t_{0}$ and $\epsilon$ as in the previous case, gives $n_{0}=171.6$ so that we would use 173 terms according to our formula. The number of terms that is actually needed in this case is around 145. Thus in this case again our formula gives too many terms, but not by as large a ratio as in the previous case.

\section{APPENDIX}

The method under discussion is based on the expansion of $\bar{F}(S)$ around the point at infinity. Thus we substitute $1 / Z$ for $S$ and get

$$
f(Z)=\bar{F}\left(\frac{1}{Z}\right)=\frac{Z\left\{A_{0}{ }^{0}+A_{1}{ }^{0} Z+\cdots+A^{0}{ }_{N-1} Z^{N-1}\right\}}{1+b_{1} Z+\cdots+b_{N} Z^{N}} .
$$

Next we write

$$
f(Z)=Z g(Z)
$$


so

$$
g(Z)=\frac{p_{0}(Z)}{Q(Z)}
$$

where

$$
p_{0}(Z)=A_{0}{ }^{0}+A_{1}{ }^{0} Z+\cdots+A^{0}{ }_{N-1} Z^{N-1}
$$

and

$$
Q(Z)=1+b_{1} Z+\cdots+b_{N} Z^{N} .
$$

Now $g(Z)$ is analytic at the origin $(Z=0)$, and so it has a MacLaurin series:

$$
g(Z)=\alpha_{0}+\alpha_{1} Z+\alpha_{2} Z^{2}+\cdots .
$$

We shall show presently that these coefficients $\alpha_{j}$, are the same as the $\alpha_{j}$ in equation (3), and also that the series (3) converges for all values of $t$.

To evaluate the $\alpha_{j}$ in equation (13) we use the method of [1], which becomes somewhat simpler in this case since we are expanding around the origin. From (10) and (13) we have

$$
p_{0}(Z)=Q(Z)\left[\alpha_{0}+\alpha_{1} Z+\alpha_{2} Z^{2}+\cdots\right] .
$$

Then

$$
\alpha_{0}=\frac{p_{0}(0)}{Q(0)}=A_{0}{ }^{0} .
$$

Now, having $\alpha_{0}$, we note that

$$
p_{0}(Z)-\alpha_{0} Q(Z)
$$

has 0 as a root, so we can define another polynomial

$$
p_{1}(Z)=\frac{p_{0}(Z)-\alpha_{0} Q(Z)}{Z}
$$

Then

$$
p_{1}(Z)=A_{0}^{1}+A_{1}^{1} Z+A_{2}^{1} Z^{2}+\cdots+A^{1}{ }_{N-1} Z^{N-1}
$$

and the coefficients $A_{k}{ }^{1}$ are given by equations (4). We then have

$$
p_{1}(Z)=Q(Z)\left[\alpha_{1}+\alpha_{2} Z+\alpha_{3} Z^{2}+\cdots\right] \text {. }
$$

Thus we get

$$
\alpha_{1}=\frac{p_{1}(0)}{Q(0)}=A_{0}^{1}
$$

Then defining the polynomial

$$
p_{2}(Z)=\frac{p_{1}(Z)-\alpha_{1} Q(Z)}{Z},
$$


we get

$$
p_{2}(Z)=A_{0}^{2}+A_{1}^{2} Z+\cdots+A^{2}{ }_{N-1} Z^{N-1}
$$

where the coefficients $A_{1}{ }^{2}$ are given by equations (5). Then $\alpha_{2}=A_{0}{ }^{2}$. If we continue in this manner we get the iterative relationships (6).

Next we shall use the method of [2] to prove equation (3), since this will assist us in obtaining an error criterion. Suppose $\epsilon$ is a small positive number. We want to show that in a given interval, $0-t_{0}$, we can choose $n_{0}$ such that when $n>n_{0}$,

$$
\left|F(t)-\sum_{j=0}^{n} \alpha_{j} \frac{t^{i}}{j !}\right|<\epsilon, \quad 0<t_{0} \leq t_{0}
$$

First let $r_{1}$ be a positive number such that all the roots of $Q(Z)$ are greater than $r_{1}$ in absolute value. Then $g(Z)$ is analytic for $|Z| \leq r_{1}$, and so also is

$$
h(Z)=\frac{p_{1}(Z)}{Q(Z)}
$$

From equation (18) we see that

$$
h(Z)=\sum_{j=1}^{\infty} \alpha_{j} Z^{j-1} .
$$

The series (22) will converge uniformly for $|Z| \leq r_{1}$. Thus if we let

$$
R_{n}(Z)=\sum_{j=n+1}^{\infty} \alpha_{j} Z^{j-1}
$$

then for any positive number $\epsilon_{1}$ we can find $n_{0}\left(\epsilon_{1}\right)$ such that $\left|R_{n}(Z)\right|<\epsilon_{1}$ when $|Z| \leq r_{1}$ and $n>n_{0}\left(\epsilon_{1}\right)$.

$\epsilon_{1}$ can be any positive number and we will choose

$$
\epsilon_{1}=\frac{2 \epsilon}{r_{1}} e^{-\frac{t_{0}}{r_{1}}}
$$

Since the point $\frac{1}{r_{1}}+i 0$ is to the right of all the singularities of $\bar{F}(S)$, we have by the inversion theorem

$$
2 \pi i F(t)=\int_{\frac{1}{r_{1}}-i \infty}^{\frac{1}{r_{1}}+i \infty} \bar{F}(S) e^{s t} d S=\int_{\frac{1}{r_{1}}-i \infty}^{\frac{1}{r_{1}}+i \infty} e^{s t}\left\{\sum_{j=0}^{\infty} \frac{\alpha_{j}}{S^{j+1}}\right\} d S .
$$

The last step follows from equations (8), (9), and (13) substituting $1 / S$ for $Z$.

Next we consider the function

$$
F_{n}(t)=\sum_{j=0}^{n} \alpha_{j} \frac{t^{j}}{j !}
$$


The Laplace transform of $t^{i} / j !$ is $\frac{1}{S^{j+1}},(j=0,1,2, \cdots)$.

Thus by the inversion theorem

$$
2 \pi i F_{n}(t)=\sum_{j=0}^{n} \alpha_{j}\left[\int_{\frac{1}{r_{1}}-i \infty}^{\frac{1}{r_{1}}+i \infty} \frac{e^{s t}}{S^{j+1}} d S\right]
$$

Then for $n>n_{0}\left(\epsilon_{1}\right)$, we have

$$
\begin{aligned}
& \left|F(t)-F_{n}(t)\right| \\
& =\frac{1}{2 \pi}\left|\int_{\frac{1}{r_{1}}-i \infty}^{\frac{1}{r_{1}}+i \infty} e^{s t} \sum_{j=0}^{\infty} \frac{\alpha_{j}}{S^{j+1}} d S-\sum_{j=0}^{n} \alpha_{j} \int_{\frac{1}{r_{1}}-i \infty}^{\frac{1}{r_{1}}+i \infty} \frac{e^{s t}}{S^{j+1}} d S\right| \\
& =\frac{1}{2 \pi}\left|\int_{\frac{1}{r_{1}}-i \infty}^{\frac{1}{r_{1}}+i \infty} e^{s t} \frac{R_{n}\left(\frac{1}{S}\right)}{S^{2}} d S\right|=\left|\int_{-\infty}^{\infty}\left\{e^{\frac{t}{r_{1}}+i y t} \frac{R_{n}\left(\frac{1}{\frac{1}{r_{1}}+i y}\right)}{\left(\frac{1}{r_{1}}+i y\right)^{2}}\right\} d y\right| \cdot \frac{1}{2 \pi} \\
& <\frac{\epsilon_{1} e^{\frac{t}{r_{1}}}}{2 \pi} \int_{-\infty}^{\infty} \frac{d y}{\frac{1}{r_{1}^{2}}+y^{2}}=\frac{\epsilon e^{\frac{t-t_{0}}{r_{1}}}}{r_{1} \pi}\left[r_{1} \pi\right] \leq \epsilon
\end{aligned}
$$

if $0<t \leq t_{0}$. The numbers, $t_{0}$ and $\epsilon$ may have any positive values. Once they are chosen we choose $\epsilon_{1}$ by equation (24), and then find $n_{0}\left(\epsilon_{1}\right)$. The actual finding of a number $n_{0}$, after having found $\epsilon_{1}$ is the task which we will consider next. However we have proved that the series of equation (3) converges to $F(t)$ for all positive $t$. Also we see that the number $n_{0}$ which makes $\left|F(t)-F_{n}(t)\right|<\epsilon$ for $t=t_{0}$, also does so for all values of $t$ between 0 and $t_{0}$.

Next we ask the question: Having given $\epsilon_{1}$ from equation (24), how do we actually find a number $n_{0}$ such that $\left|R_{n}(Z)\right|<\epsilon_{1}$ when $|Z| \leq r_{1}$ and $n>n_{0}$ ? We must do this in order to have a formula for obtaining a sufficient number of terms in the series (3). Also we have not yet chosen $r_{1}$, except to say that it is less than the modulus of each of the roots of $Q(Z)$.

First of all we define

$$
r_{0}=\frac{1}{1+b}
$$

where $b$ is the maximum of the numbers $\left|b_{1}\right|,\left|b_{2}\right|, \cdots,\left|b_{N}\right|$. Then for $|Z| \leq r_{0}$,

$$
\begin{aligned}
|Q(Z)| \geq 1-b\left[\frac{1}{1+b}+\right. & \left.\left(\frac{1}{1+b}\right)^{2}+\cdots+\left(\frac{1}{1+b}\right)^{N}\right] \\
& =1-\left(\frac{b}{1+b}\right) \frac{1-\left(\frac{1}{1+b}\right)^{N}}{1-\left(\frac{1}{1+b}\right)}=\frac{1}{(1+b)^{N}} .
\end{aligned}
$$


Thus all the roots of $Q(Z)$ are outside the circle $|Z|=r_{0}$. Now we choose

$$
r_{1}=\theta r_{0}=\frac{\theta}{1+b}
$$

where $\theta$ is as yet undetermined, except that

$$
0<\theta<1 \text {. }
$$

Then for $|Z| \leq r_{1}$, we have

$$
R_{n}(Z)=\sum_{j=n+1}^{\infty} \alpha_{j} Z^{j-1}=h(Z)-\sum_{j=1}^{n} \alpha_{j} Z^{j-1}
$$

Also

$$
\alpha_{j}=\frac{h^{(j-1)}(0)}{(j-1) !}
$$

where $h^{(k)}(Z)$ is the $k^{\text {th }}$ derivative of $h(Z)$. From Cauchy's integral formula, we have

$$
h(Z)=\frac{1}{2 \pi i} \oint_{c_{0}} \frac{h(w)}{w-Z} d w
$$

and

$$
h^{(k)}(Z)=\frac{k !}{2 \pi i} \oint_{c_{0}} \frac{h(w)}{(w-Z)^{k+1}} d w
$$

if $|Z| \leq r_{1}$, where $C_{0}$ is the circle $|w|=r_{0}$.

Thus, for $|Z| \leq r_{1}$,

$$
R_{n}(Z)=\frac{1}{2 \pi i} \oint_{c_{0}} \frac{h(w)}{w-Z} d w-\sum_{j=1}^{n} \frac{Z^{j-1}}{2 \pi i} \oint_{c_{0}} \frac{h(w)}{w^{j}} d w .
$$

The second term

$$
\begin{aligned}
& \sum_{j=1}^{n} \frac{Z^{j-1}}{2 \pi i} \oint_{c_{0}} \frac{h(w)}{w^{j}} d w \\
& =\frac{1}{2 \pi i} \oint_{c_{0}} \frac{h(w)}{w} \sum_{j=1}^{n}\left(\frac{Z}{w}\right)^{j-1} d w=\frac{1}{2 \pi i} \oint_{c_{0}} \frac{h(w)}{w} \frac{\left(1-\frac{Z}{w}\right)^{n}}{\left(1-\frac{Z}{w}\right)} d w \\
& =\frac{1}{2 \pi i} \oint_{c_{0}} \frac{h(w)}{w-Z} d w-\frac{1}{2 \pi i} \oint_{c_{0}} \frac{h(w)}{(w-Z)}\left(\frac{Z}{w}\right)^{n} d w .
\end{aligned}
$$

Hence

$$
R_{n}(Z)=\frac{1}{2 \pi i} \oint_{c_{0}} \frac{h(w)}{(w-Z)}\left(\frac{Z}{w}\right)^{n} d w
$$


Thus, since $|Z| \leq r_{1}=\theta r_{0}$,

$$
\left|R_{n}(Z)\right| \leq \frac{1}{2 \pi} \frac{M}{r_{0}(1-\theta)} \theta^{n} 2 \pi r_{0}=\frac{M \theta^{n}}{(1-\theta)}
$$

where $M$ is the maximum of the function $|h(w)|$ on the circle $C_{0}$.

On the circle $C_{0}$, we have

$$
|h(w)|=\frac{\left|p_{1}(w)\right|}{|Q(w)|} \leq(1+b)^{N}\left|p_{1}(w)\right|
$$

using (30).

The coefficients $A_{k}{ }^{1}$ of $p_{1}(Z)$ are given by equations (4), and since $\alpha_{0}=A_{0}{ }^{0}$, we see from these equations that

$$
\left|A_{k}{ }^{1}\right| \leq A(1+b), \quad k=0,1, \cdots,(N-1),
$$

where $A$ is the maximum of the numbers $\left|A_{0}{ }^{0}\right|,\left|A_{1}{ }^{0}\right|, \cdots,\left|A^{0}{ }_{N-1}\right|$.

Thus on the circle $C_{0}$,

$$
\begin{array}{r}
\left|p_{1}(w)\right| \leq A(1+b)\left[1+\frac{1}{1+b}+\left(\frac{1}{1+b}\right)^{2}+\cdots+\left(\frac{1}{1+b}\right)^{N-1}\right] \\
=A(1+b) \frac{1-\left(\frac{1}{1+b}\right)^{N}}{1-\frac{1}{1+b}}
\end{array}
$$

Therefore, using (40),

$$
M \leq A(1+b)^{N+1} \frac{1-\left(\frac{1}{1+b}\right)^{N}}{1-\frac{1}{1+b}}=\frac{A(1+b)^{2}\left[(1+b)^{N}-1\right]}{b} .
$$

Thus, returning to condition (39) we see that for $|Z| \leq r_{1}$,

$$
\left|R_{n}(Z)\right| \leq \frac{A(1+b)^{2}\left[(1+b)^{N}-1\right] \theta^{n}}{b(1-\theta)}
$$

Thus, we wish to have

This will be true if

$$
\frac{A(1+b)^{2}\left[(1+b)^{N}-1\right] \theta^{n}}{b(1-\theta)}<\epsilon_{1} .
$$

$$
\left(\frac{1}{\theta}\right)^{n}>\frac{A(1+b)^{2}\left[(1+b)^{N}-1\right]}{b \epsilon_{1}(1-\theta)},
$$

that is,

$$
n>\frac{\ln \left[\frac{A(1+b)^{2}\left[(1+b)^{N}-1\right]}{b \epsilon_{1}(1-\theta)}\right]}{\ln \left(\frac{1}{\theta}\right)} .
$$


Thus we have found a number $n_{0}\left(\epsilon_{1}\right)$ which has the required property, namely

$$
n_{0}=\frac{\ln \left[\frac{A(1+b)^{2}\left[(1+b)^{N}-1\right]}{b \epsilon_{1}(1-\theta)}\right]}{\ln \left(\frac{1}{\theta}\right)} .
$$

In order to relate $\epsilon_{1}$ to $\epsilon$, the desired maximum error in $F(t)$, and the interval $\left(0\right.$ to $\left.t_{0}\right)$, we use equations (24) and (31), and get

$$
\begin{aligned}
n_{0} & =\frac{\ln \left[\frac{\left.A(1+b)\left[(1+b)^{N}-1\right] \theta e^{\frac{(1+b) t_{0}}{\theta}}\right]}{2 b(1-\theta) \epsilon}\right]}{\ln \left(\frac{1}{\theta}\right)} \\
& =\frac{(1+b) t_{0}}{\theta \ln \left(\frac{1}{\theta}\right)}+\frac{\ln \left[\frac{A(1+b)\left[(1+b)^{N}-1\right] \theta}{2 b(1-\theta) \epsilon}\right]}{\ln \left(\frac{1}{\theta}\right)} .
\end{aligned}
$$

We still have not chosen $\theta$, except to say that it is a positive number less than unity. We somewhat arbitrarily choose $\theta$ so as to make the $t_{0}$ term as small as possible, i.e., the value which makes $\theta \ln (1 / \theta)$ maximum. This value is

$$
\theta=e^{-1}
$$

Thus

$$
n_{0}=e(1+b) t_{0}+\ln \left[\frac{A(1+b)\left[(1+b)^{N}-1\right]}{2 b \epsilon(e-1)}\right]
$$

which is the same as equation (7).

This paper was written while the author was employed by the MinneapolisHoneywell Regulator Company of Minneapolis, Minnesota. The author wishes to express his appreciation to Mr. Eric Clamons of Minneapolis-Honeywell, for many suggestions, made by him, pertaining to this work.

\section{P. Flemming}

Canadian Armament Research and

Development Establishment

Valcartier, Quebec.

1. D. P. Flemming, "An iterative method for Taylor expansion of rational functions and applications," to appear in MTAC.

2. H. S. CARSLAW \& J. C. JAEGER, Operational Methods in Applied Mathematics, 2nd Edition, Oxford University Press, London, 1948, Chapter XIII. 